\title{
A Field Concept of the Black Hole
}

\author{
Ogaba Philip Obande ${ }^{1}$ \\ ${ }^{1}$ Department of Chemistry, Ahmadu Bello University, Zaria, Nigeria (Retired) \\ Correspondence: Ogaba Philip Obande, Department of Chemistry, Ahmadu Bello University, Zaria, Nigeria \\ (Retired). E-mail: gababands@gmail.com
}

Received: May 3, 2018

doi:10.5539/apr.v10n4p66
Accepted: May 24, 2018

Online Published: July 16, 2018

URL: https://doi.org/10.5539/apr.v10n4p66

\begin{abstract}
A new perspective of the black hole BH is introduced. Based on the assumption that space and matter are fundamentally fields and governed by same deterministic quantum laws, the field dynamics is analyzed with classical (Newtonian) mechanics to argue that: i) the BH is not a singularity but Hawking's 'apparent horizon'; ii) the Schwarzschild BH does not exist; iii) the naked 'singularity' NS might not be ruled out in observational atomic and molecular emissions; iv) the Kerr-Newman black hole KBH functions to modulate and transform frequency of matter waves and re-orient same across the three particle-generations universes in perpetual energy re-cycles, no new creation but endless energy re-cycling; vi) the $\mathrm{BH}$ process is not thermodynamics but electrodynamics; vii) accretion is not random, it is motivated by a universal 'aging' process in which matter progresses from one symmetry group to another; viii) spatial alignment arises from universal diagonal orientation of constituent elements of intrinsic cubic geometry of nature's periodic envelopes; ix) all spatial periodic envelopes are binaries, the causality is illustrated in some detail. It is suggested that a frequency modulator/transformer circuitry might be a better model for simulating the BH than thermodynamics.
\end{abstract}

Keywords: binary star, black hole, cosmological parameters, dark matter, field concept, galactic nucleus

\section{Introduction}

Experimental physics has produced visual evidence of the atom's intrinsic wave-particle duality (WPD), see the Pan-STARRS1 'Static Sky', New Atlas (2016) and our interpretation of the image, Obande (2017a). It implies dual nature not only of the atom but also of reality itself and exposes inherent limitations of the reigning physics paradigm to fully describe nature with clear distinction between attributes of each of the two composite forms. The WPD engenders a reality that differs markedly from established notion, it reveals an objective reality comprising a wave (bosonic) field in which three particulate (fermionic) matter fields are encased, the four fields are mutually orthogonal. The structure replicates the solid, liquid, and gaseous phases of matter, in this case they correspond to a bosonic field in 'equilibrium' with three integral fermionic fields responsible for observational three 'particle-generations'. A mono-phasic spacetime model therefore grossly underestimates reality. Spacetime is, if you like, 'quadri-phasic.' If you, therefore, seek to model nature with, say, time-density fluctuation, the existing formalism will require at least two equations of state, one for the boson and another for the fermion fields; indeed, a rigorous approach would require four equations-of-state, one for the boson and one for each of the three fermion fields. The four fields define with common chemical periodicity, Obande (2015a, 2016a). Since one of these fields gives rise to our visible reality there is no immediate reason to preclude the other fields from producing similar realities, we therefore attribute each field to a universe; notably, they are co-existent, inseparable, interactive and contribute to a common experience of single existence. Every object defines with all four fields; they are not parallel universes but ref. frames of a single cosmic reality, we label them $\mathrm{U}_{\mathrm{w}}{ }_{\mathrm{w}} ; \mathrm{U}^{*}{ }_{\mathrm{p}}$; $\mathrm{U}_{\mathrm{p}}^{\mathrm{o}}$ and $\mathrm{U}_{\mathrm{p}}^{\prime}$ to denote respectively the Cosmic Vacuum Field or Absolute Universe; particulate Analogue of the Absolute; our Visible Universe and its Invisible Conjugate Universe. Collectively, $\mathrm{U}_{\mathrm{p}}^{*}, \mathrm{U}_{\mathrm{p}}{ }_{\mathrm{p}}$ and $\mathrm{U}_{\mathrm{p}}^{\prime}$ constitute the three particlegenerations universes PGUs, each characterized with specific mass-energy $\mathrm{m}$-e values of the chemical periodicity; it accounts for each element having four atomic mass values $\mathrm{m}$, electromagnetic e-m oscillation frequencies $\vartheta$, and internal energies E, Obande (2015a). The notion is by no means new to physics, the difference is that the three particle-generations now factor seamlessly into the formalism and we now know their origin. 


\subsection{Quantized Space, Mass and Energy}

The field approach takes the position that vacuous space is simultaneously quantized with matter; indeed, fundamental quantum spacetime, $\vartheta=1 \mathrm{~s}^{-1}$, determines unit quantum energy, $\mathrm{h} \vartheta$, and mass $\mathrm{h} \vartheta / \mathrm{c}^{2}$. In matter the unit corresponds to the electron with $\vartheta_{\mathrm{e}(\mathrm{w})}=1 \mathrm{~Hz} \equiv \mathrm{E}_{\mathrm{e}(\mathrm{w})}=6.62607 \times 10^{-34} \mathrm{~J} ; \mathrm{m}_{\mathrm{e}(\mathrm{w})}=7.3725 \times 10^{-51} \mathrm{~kg}$ and $\mathrm{m}_{\mathrm{e}(\mathrm{p})}=$ $4.8828125 \times 10^{-7} \mathrm{~kg}$, Obande $(2015 \mathrm{a})$, where $\mathrm{m}_{\mathrm{e}(\mathrm{w})}$ and $\mathrm{m}_{\mathrm{e}(\mathrm{p})}$ refer to electron wave and particle rest mass respectively. Strict compliance with convention therefore requires the Planck scale to read: $\mathrm{T}_{\mathrm{P \ell}}=1 \mathrm{~s}$; $\mathrm{L}_{\mathrm{P} \ell}=\mathrm{c}_{\mathrm{o}} / \mathrm{T}_{\mathrm{Pl}}=2.9979 \times 10^{8} \mathrm{~m} ; \mathrm{M}_{\mathrm{P} \ell}=7.3725 \times 10^{-51} \mathrm{~kg} ; \mathrm{E}_{\mathrm{P} \ell}=6.6261 \times 10^{-34} \mathrm{~J}\left(\right.$ check: $\left.\mathrm{E}_{\mathrm{P} \ell}=\mathrm{M}_{\mathrm{P} \ell} \times \mathrm{L}_{\mathrm{P} \ell}^{2}=\mathrm{mc}^{2}\right)$, any lower or higher dimension is not a quantum, i.e., 'smallest whole', but a fraction or multiple; literature's Planck dimensions serve academic purposes but do not relate to reality.

\subsection{Mass-Energy Equivalence: Astrophysical and Cosmological Implications}

Identical quantum behavior and interactions (reactions) define matter and space; notably, both share common quantum energy coefficient, $\mathrm{h}=6.62607 \times 10^{-34} \mathrm{~J} \mathrm{~s}$, Obande (2015a) thus, the atom, condensed matter, and 'vacuum' space all analyze with same classical SHM formalism. Astrophysics is getting increasingly acquainted with the spatial quantum, often referred to as 'periodic box', e.g., Conselice et al. (2016). The key point is that with use of relevant parameters, classical (Newtonian) physics applies equally across spatial and matter periodicities; furthermore, matter and spatial quanta 'react' alike, the major determinant being geometric in character, Obande (2017c). Nature makes no distinction between wave and particle in its primitive interactions, all is wave, Born (1935), Macken (2011), Hobson (2013), Obande (2013), Consiglio (2015, 2016). The present (classical) approach points to existence of a spatial periodicity analogous to the chemical (matter) periodicity space is defined with same quantum effects and chemical physics as matter complete with hierarchical quanta sizes that parallel atomic mass evolution. The new picture facilitates an incredibly simple but much more analytically powerful theoretical approach; it is observational, absolutely free of singularities (hence this investigation) and most analyses reduce to intra- and inter-ref. frame energy correlations. We introduce the periodic background of the $\mathrm{BH}$ phenomenon.

\subsubsection{On the 5th Matter/Spatial Periodic Group}

Nature's periodicity NP of the chemical elements comprises nine periods and nine groups, Obande (2017c), it differs from the conventional 8-group structure in existence of a central group zero ' 0 ' which separates conventional ' $\mathrm{A}$ ' and ' $\mathrm{B}$ ' blocks of elements. This central group 0 or group 5 is crucial to the field concept of the $\mathrm{BH}$, it centers conventional group IV to give the periodic order: $0,+\mathrm{I},+\mathrm{II},+\mathrm{III},+\mathrm{IV}, 0,-\mathrm{IV},-\mathrm{III},-\mathrm{II},-\mathrm{I}, 0$; positive and negative \pm signs, of course, denote electrostatic charges; in reality, however, they refer to contrary angular momentum vector orientations, Obande (2017c). The noble gas, as usual, belongs to group zero, however, NP's central group ' 0 ' refers to group IV's epicenter, i.e., $+4,0$, -4 . Unlike the noble gas, central group ' 0 ' is a unique vectorial transition zone which, as we shall see presently, manifests the BH phenomenon.

\section{Procedure}

Using $\vartheta$ or $\mathrm{m}$ in $\mathrm{h} \vartheta=\mathrm{mc}^{2}$ we simply correlate the chemical element's internal (atomic) energy values across the three particle-generations universes PGUs $\mathrm{U}^{*}{ }_{\mathrm{p}}, \mathrm{U}_{\mathrm{p}}^{\mathrm{p}}$, and $\mathrm{U}_{\mathrm{p}}^{\prime}$; the details have been reported severally, Obande (2015a, 2016b).

\section{Results}

Mass-energy matrix flux across the PGUs is investigated with simple graphical correlation of the chemical element's internal energy $\vartheta$ or m value. The results are presented in seven figures, one flowchart and three tables. Figure 1 is a $\log -\log$ plot of $\vartheta_{p}^{\prime}$ vs. $\vartheta^{*}$; Figure 2 is another $\log -\log$ plot of $\vartheta_{p}^{\circ}$ vs. $\vartheta_{p}^{\prime}$; Figure 3 is $\log$-lin plot of $\vartheta^{x}{ }_{p}$ vs. $Z_{\mathrm{n}}$ where $\mathrm{x}$ denotes the ref. frame and $Z_{\mathrm{n}}$ is atomic number in nature's periodicity $\mathrm{NP}$ of the chemical elements, Obande (2017c); Figure 4 is a plot of the ratio $\mathrm{m}_{\mathrm{p}}^{\mathrm{x}} / \mathrm{m}^{*} \mathrm{p}$ vs. $Z_{\mathrm{n}}$; Figs. 5 and 6 show locations of relevant objects in ellipsoidal morphology of the spatial periodic envelope and Figure 6a is a sketch of the primitive vortical electrical pair of the universal quantum envelope. Spacetime matrix flux between the visible universe $\mathrm{U}_{\mathrm{p}}^{\circ}$ and its invisible conjugate $\mathrm{U}_{\mathrm{p}}^{\prime}$ is summarized in a flowchart. Table 1 provides $\vartheta$ values of periodic elements relevant to the $\mathrm{BH}$ process; Table 2 differentiates between $m$ and $\vartheta$ values in $\mathrm{U}_{\mathrm{p}}^{\mathrm{o}}$ and $\mathrm{U}_{\mathrm{p}}^{\prime}$ for some elements to illustrate effect of the $\mathrm{BH}$ process on spacetime matrices of the two ref. frames.

\subsection{Quantification of the Black Hole Process}

Figure 1 reveals an inflexion in the $U_{p}^{\prime} \leftrightarrow U^{*}$ matrix flux centered at periodic group 5, similar m-e exchange between $U_{p}^{o}$ and $U_{p}^{\prime}$ in Figure 2 reveals a bimodal flux gradient. As shown in Figure 3, variation of atomic energy with an increase in atomic number $Z_{\mathrm{n}}$ reproduces in $\vartheta_{\mathrm{p}}^{\prime}$ the inflexion observed in Figure 1. Reduction of $\mathrm{U}_{\mathrm{p}}^{*}, \mathrm{U}_{\mathrm{p}}^{o}$ and $U_{p}^{\prime}$ energy values to corresponding $U^{*}{ }_{p}$ values in Figure 4 highlights the causal agent of the inflexion. We 
attribute the inflexion and other changes in matrix flux at group 5 to the black hole process, it implicates spacetime matrix exchange across the four states of nature: $U^{*}{ }_{w} \leftrightarrow U^{*}{ }_{p} \leftrightarrow U_{p}^{o} \leftrightarrow U_{p}^{\prime}$, more specifically, the observational process transforms visible $\vartheta_{\mathrm{p}}^{\circ}$ to invisible matrices $\vartheta_{\mathrm{p}}^{\prime}$. In other words, accretion transforms the particulate atom from a visible lower potential energy form to an invisible but much higher energy particulate form through an upgrade of $\vartheta_{\mathrm{p}}^{\circ}$ to corresponding $\vartheta_{\mathrm{p}}^{\prime}$ value, e.g., we have for $\mathrm{Kr}$, in Table 1 the transformation $\vartheta_{\mathrm{p}}^{\circ}=1.746 \times 10^{5} \mathrm{~Hz}$ to $\vartheta_{\mathrm{p}}^{\prime}=4.092 \times 10^{6} \mathrm{~Hz}$ and corresponding rest mass upgrade from visible $\mathrm{m}_{\mathrm{p}}^{\mathrm{o}}=0.0838 \mathrm{~kg} \mathrm{u}^{-1}$ to invisible $\mathrm{m}_{\mathrm{p}}^{\prime}=$ $1.964 \mathrm{~kg} \mathrm{u}^{-1}$. The subject presents a curious scenario, for instance, it raises the question, why does visible matter become invisible when the constituent atoms become electrically charged and where does the extra rest mass (2344\%!) come from? It reveals that the black hole transforms visible neutral matter to invisible charged particles to effect mass-energy flux circulation through the four phases of reality. We deduce as follows from the results: The black hole/process: i) is not a hole, it is a spatial periodic envelope in which field angular momentum vector orientation of the periodic element's spin changes sign, i.e., an inflexion zone; thus, for matter (chemical) periodicity, the valences $0, \pm \mathrm{I}, \pm \mathrm{II}, \pm \mathrm{III}, \pm \mathrm{IV}, 0$ translate to vector orientations: $0, \rightarrow, \rightarrow, \rightarrow, \rightarrow, 0, \leftarrow, \leftarrow, \leftarrow, \leftarrow$, 0 , or, $0,1,2,3,4,0,-4,-3,-2,-1,0$, where, in matter, the central zero is the atomic nucleus (or 'gap', Oldershaw, 1989) but the 'black hole' in spatial periodicity; the arrow, of course, indicates field vector handedness or electrostatic charge; ii) is not a singularity, it is an 'engineered' segment of vacuum space designed to effect the multiple processes of frequency modulation, transformation and re-orientation to facilitate inter-ref. frame spacetime matrix exchange; iii) results in bifurcation of the visible relative to invisible particle-generations universes PGUs thus; from e to Na we have, Obande (2015a):

and from $\mathrm{Mg}$ to the end of the periodicity,

$$
\vartheta_{\mathrm{p}}^{\mathrm{o}}=\vartheta_{\mathrm{p}}^{*}+\vartheta_{\mathrm{p}}^{\prime}
$$

$$
\vartheta_{\mathrm{p}}^{\mathrm{o}}=\vartheta_{\mathrm{p}}^{*}-\vartheta_{\mathrm{p}}^{\prime}
$$

It gives rise to $\mathrm{U}_{\mathrm{p}}^{\mathrm{o}}$ 's bimodal matrix flux gradient $\vartheta_{\mathrm{p}}^{\circ} / \mathrm{Z}_{\mathrm{n}}$ of Figs. 2 and 3 and results in proximate linear evolution of empirical atomic mass, Khazman (2012), Obande $(2013,2016 \mathrm{a})$; iv) upgrades $\vartheta_{\mathrm{p}}^{\circ}$ to $\vartheta_{\mathrm{p}}^{\prime}$ values with an intensity that increases with an increase in $Z_{\mathrm{n}}$, producing an atomic mass differential of $10^{4}$ between the visible and invisible phases of the atom at the periodicity's end, e.g., we have for $A m, m_{p}^{\circ}=0.234 \mathrm{~kg} \mathrm{u}^{-1}$ and $\mathrm{m}_{\mathrm{p}}^{\prime}=3.146 \times 10^{3} \mathrm{~kg} \mathrm{u}^{-1}$. It results in much denser invisible spacetime thus, visible matter 'floats' in much denser combined matrices of invisible fermion fields or 'dark' matter/energy. Observe that the visible-invisible frequency 'upgrade' $\vartheta^{\circ} \mathrm{p}$ $\rightarrow \vartheta_{\mathrm{p}}^{\prime}$ leads to spontaneous radioactivity at At, $\vartheta^{*}{ }_{\mathrm{w}}=1.007 \times 10^{9} \mathrm{~Hz}$. Interestingly, however, radioactivity is initiated by the boson $\vartheta_{w}$ not fermion $\vartheta_{p}$ field, it is motivated by the intrinsic bosonic field centripetal acceleration encasing the fermion field, see Obande $(2015 \mathrm{~b}, 2017 \mathrm{~b})$, the subject is quite subtle. Notably, the present classical BH picture tallies with Hawking's (2014) 'apparent horizon'; instead of a singularity, theory reveals a lengthy inflexion in spacetime flux spanning conventional periods 2 to 3, i.e., Li to Ar. In summary, the results reveal that: i) the $\mathrm{BH}$ effects pseudo-linear empirical atomic mass $\mathrm{m}_{\mathrm{p}}^{\mathrm{o}}$ evolution; ii) spacetime matrix re-cycling; and iii) much denser invisible ('dark') than visible particulate matter. An attempt is made in Figures 5 and 6 to situate the BH in Nature's ellipsoidal periodic envelope.

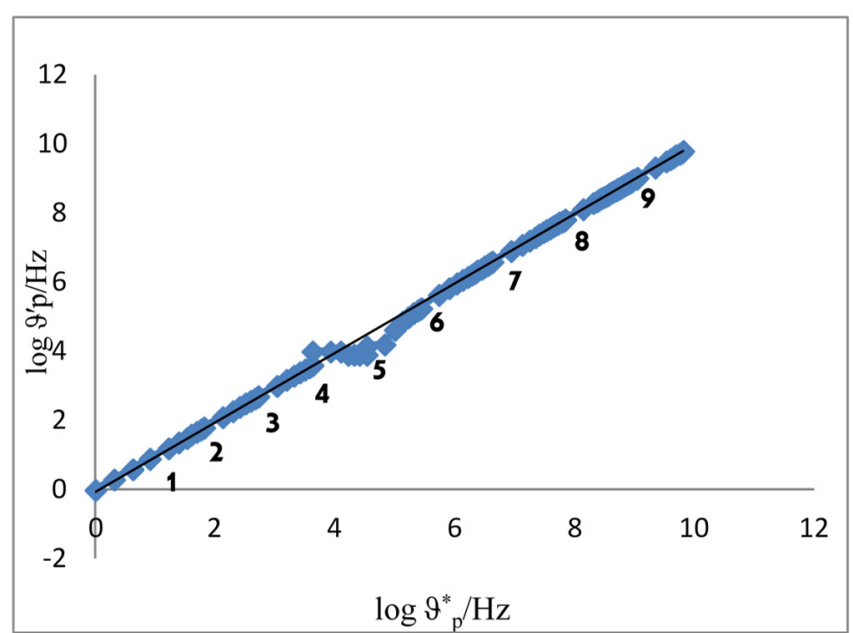

Figure 1. Plot of log $\vartheta^{\prime}$ p vs. $\vartheta^{*} p$

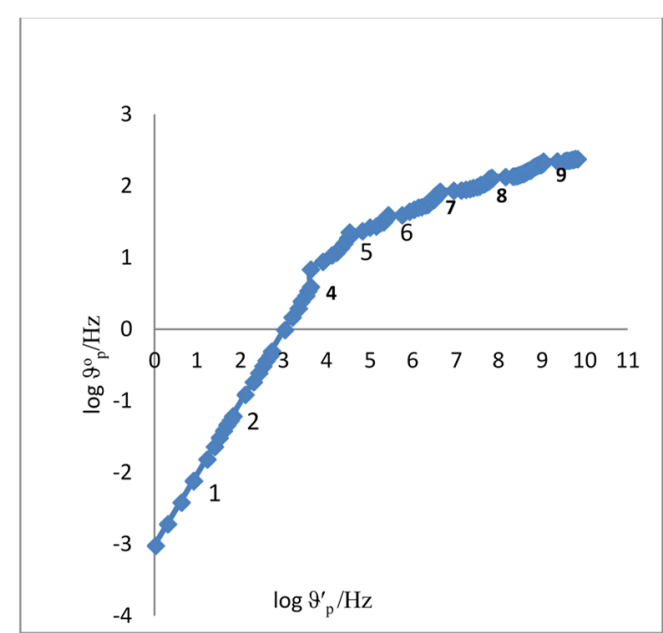

Figure 2. Plot of log Ұop vs. $\log \vartheta^{\prime} \mathrm{p}$ 


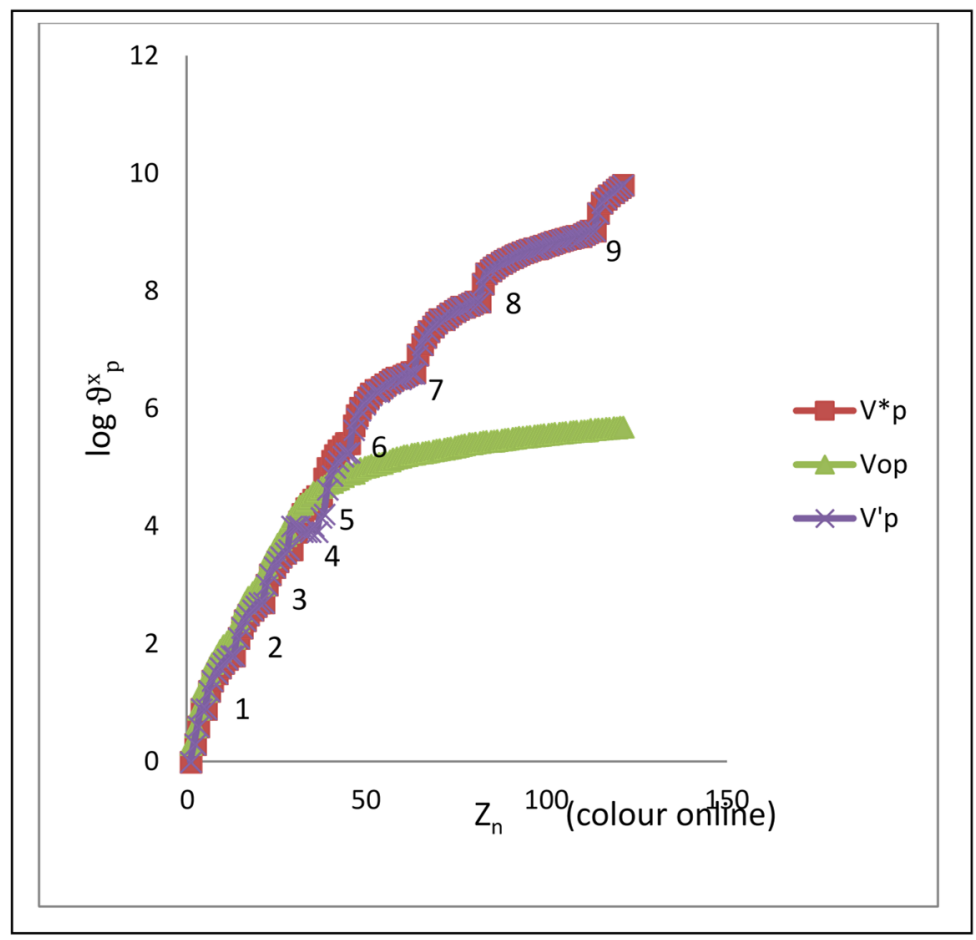

Figure 3. Plot of $\log 9 x p$ vs. Zn

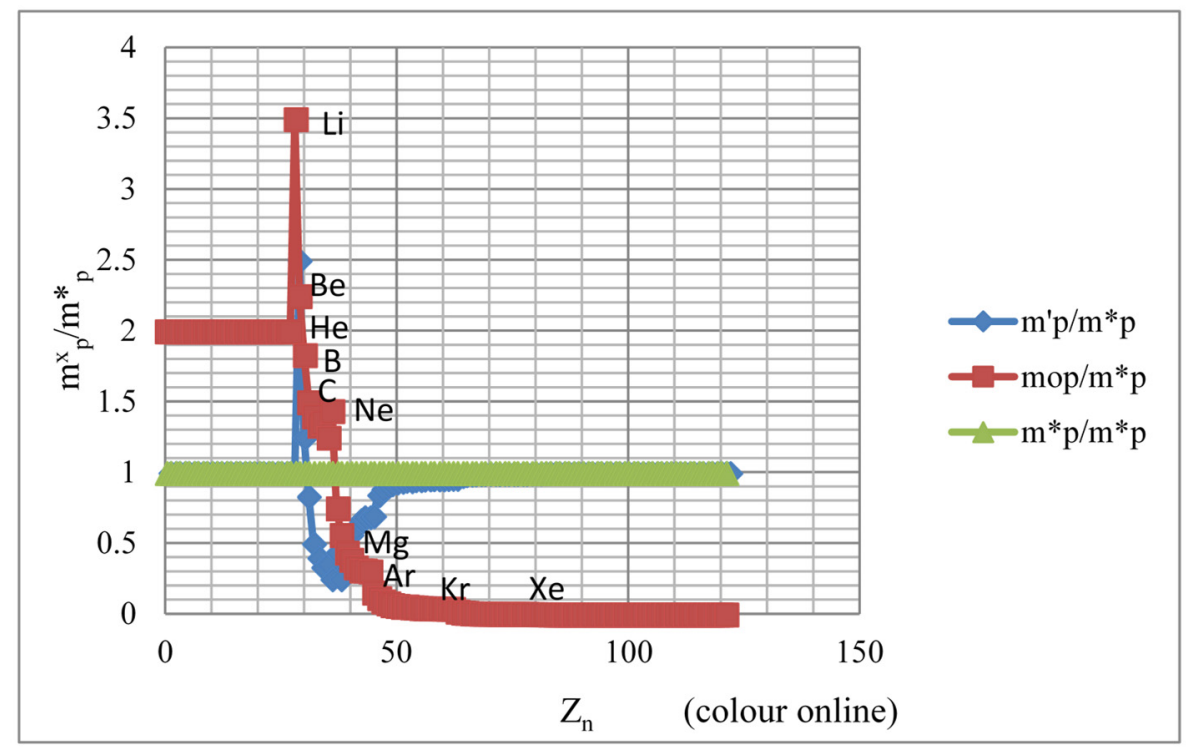

Figure 4. Plot of $m x p / m^{*} p$ vs. Zn

Table 1. Oscillation frequencies and $\mathrm{U}_{\mathrm{p}}^{\mathrm{x}} / \mathrm{U}^{*} \mathrm{p}$ ratio of some elements in $\mathrm{U}^{*} \mathrm{p}, \mathrm{U}_{\mathrm{p}}^{\mathrm{o}}$ and $\mathrm{U}_{\mathrm{p}}$

\begin{tabular}{llllllllllllllll}
\hline Element & $\mathrm{H}$ & $\mathrm{He}$ & $\mathrm{Li}$ & $\mathrm{Be}$ & $\mathrm{B}$ & $\mathrm{C}$ & $\mathrm{N}$ & $\mathrm{O}$ & $\mathrm{F}$ & $\mathrm{Ne}$ & $\mathrm{Na}$ & $\mathrm{Mg}$ & $\mathrm{Ar}$ & $\mathrm{Kr}$ \\
\hline$\vartheta_{\mathrm{p}}^{*} / \mathrm{Hz}$ & 2083 & 4167 & 4167 & 8333 & 12500 & 16666 & 20833 & 24999 & 29166 & 33332 & 33332 & 66664 & $2.7 \mathrm{E} 6$ & $4.2 \mathrm{E}+06$ \\
$\vartheta_{\mathrm{p}}^{\circ} / \mathrm{Hz}$ & 4167 & 8333 & 14458 & 18770 & 22520 & 25020 & 29187 & 33311 & 39561 & 42040 & 47894 & 50644 & $2 \mathrm{E}+05$ & $1.7 \mathrm{E}+05$ \\
$\vartheta_{\mathrm{p}}^{\prime} / \mathrm{Hz}$ & 2083 & 4167 & 10416 & 10416 & 10416 & 8333 & 8333 & 8333 & 10416 & 8333 & 14583 & 16666 & 83000 & $4.1 \mathrm{E}+06$ \\
$\vartheta_{\mathrm{p}}^{\prime} / \vartheta_{\mathrm{p}}^{*}$ & 1.00 & 1.00 & 2.50 & 1.25 & 0.83 & 0.50 & 0.40 & 0.33 & 0.36 & 0.25 & 0.44 & 0.25 & 0.30 & 0.98 & 0.98 \\
$\vartheta_{\mathrm{p}}^{\circ} / \vartheta_{\mathrm{p}}^{*}$ & 2.00 & 2.00 & 3.47 & 2.25 & 1.80 & 1.50 & 1.40 & 1.33 & 1.36 & 1.26 & 1.44 & 0.76 & 0.07 & 0.04 \\
\hline
\end{tabular}




\subsection{The Periodic Matter/Space Envelope}

The $\mathrm{BH}$ is fundamental to ellipsoidal morphology of spatial periodicities; indeed, the morphology is explicable in the context of BH-motivated inversion of the e-m transverse radiation that produces the stationary wave function associated with rest mass or energy packet. We present an understanding of the key structural elements that realize the periodic quantum envelope (Figs. 5 and 6), it is scale-invariant and cuts across the cosmic mass scale from the electron to the universe. Fig 5 is a 2D sketch of a binary system while Fig 6 is a similar 2D sketch of a 'particlegenerations' universe PGU, the symbols are defined:

\section{Figure 5: Symbols}

An open cycle locates the imponderable vacuum field, $\mathrm{U}^{*}$, it is orthogonal to the plane of the diagram.

The cycle along BD centers each periodic group in matter and in space; there are nine groups in a complete period, four on either side of a central zero, Obande (2017c). In matter the zero denotes atomic nucleus and in space it locates the black hole (see key to Figure 6a) thus, every material object is encased in and centered by the vacuum field, it accounts for 'doughnut' structure of fermions.

We identify the two explosion-like symbols at the apices B and D with the naked singularity NS or the NonRotating Black Hole NRBH. Since the box is half an envelope of the binary, only the NS/NRBH is shown, the Kerr black hole KBH locates at its diametric end as shown in Figure 6.

Observe that the gravitational field binding the couple results from the defining e-m rotational force field $\pm \mathrm{x} A / \mathrm{B}$, see Obande (2017b).

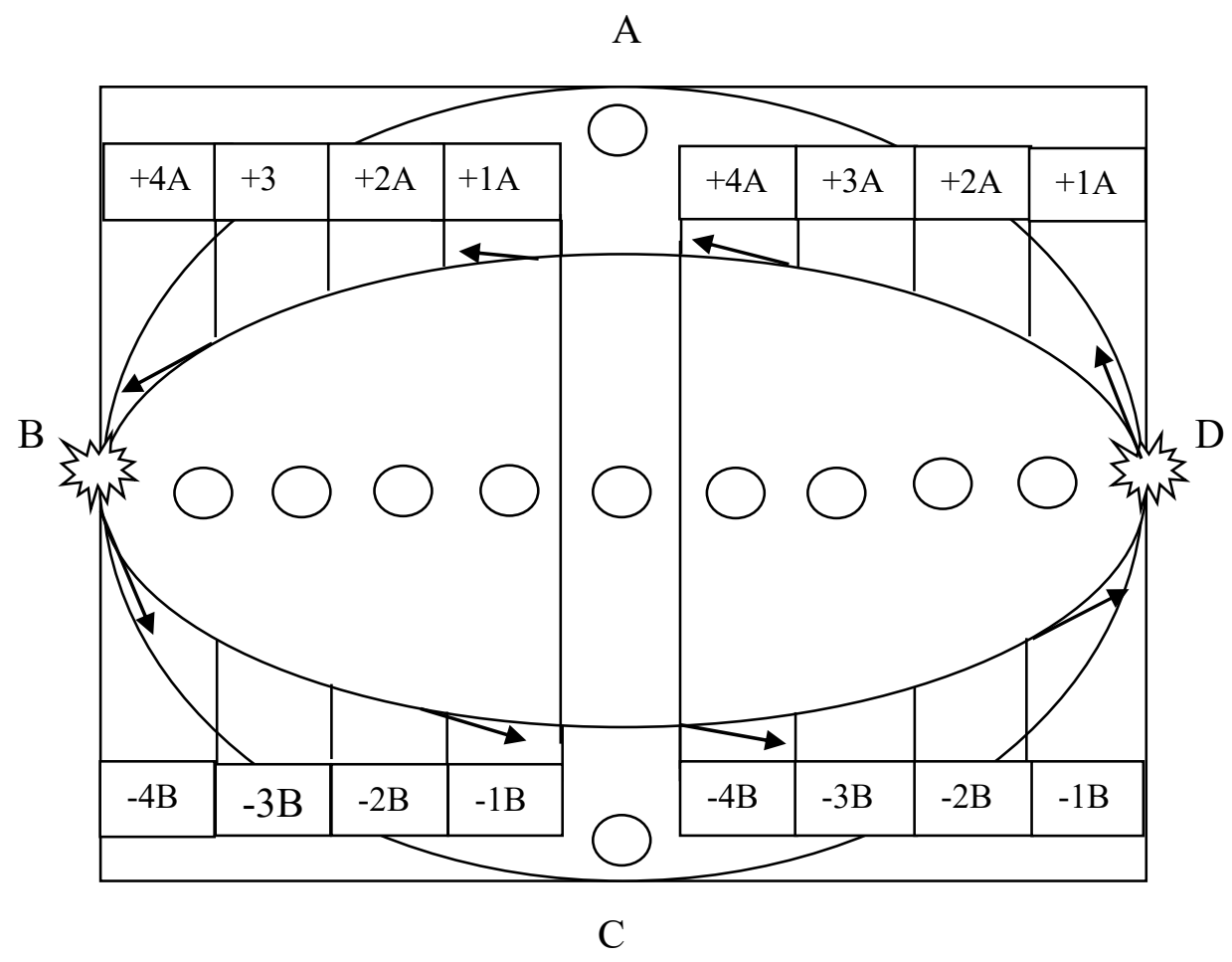

Figure 5. An illustration of a binary system using one half of an envelope to show the common binding e-m force field responsible for mutual gravitation and axial alignment of like charges responsible for mutual repulsion see Figure 6 for the symbols 


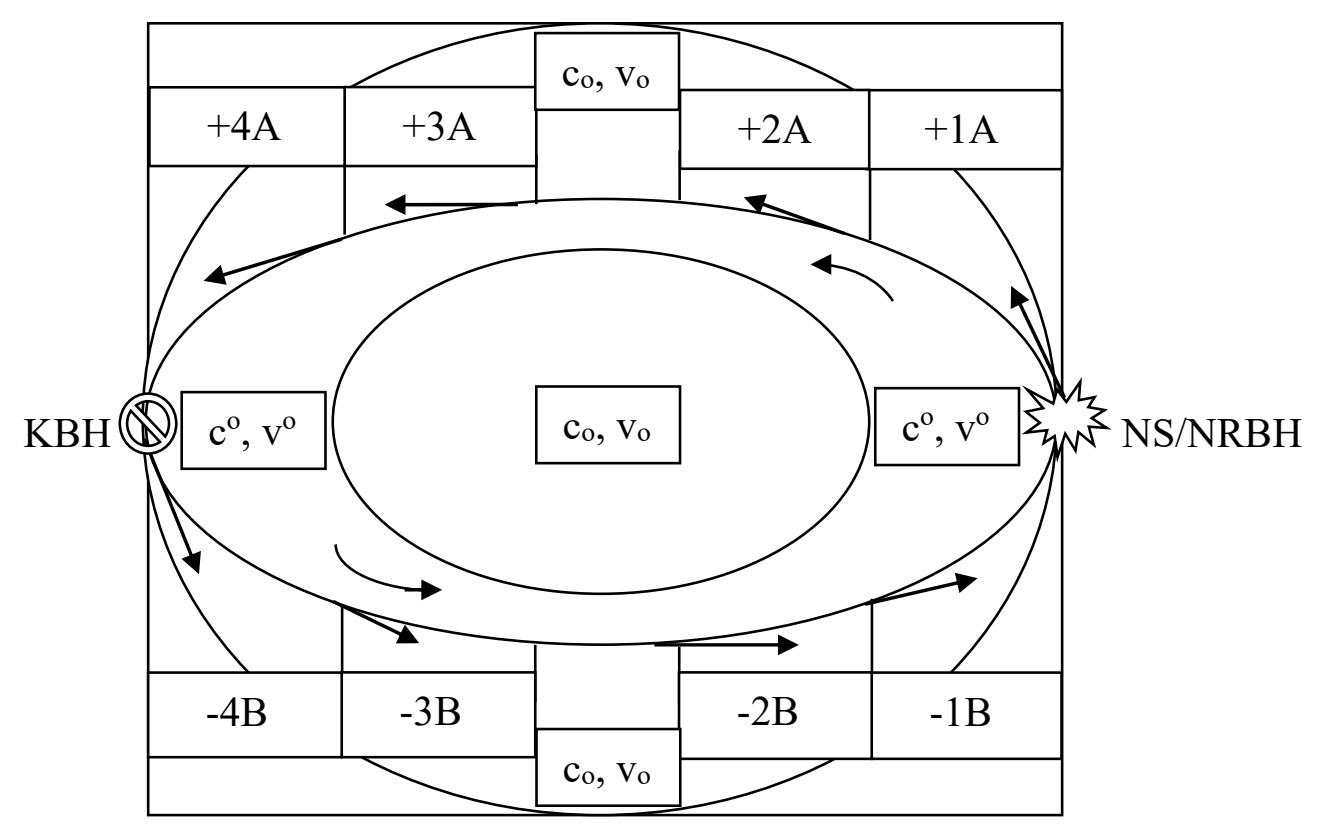

Figure 6. Schematic illustration of common characteristics of nature's material and spatial periodic envelopes

Figure 6 represents half of a binary whose twin completes, say, the $x-y$ plane, similar doublets in two other orthogonal planes, $\mathrm{x}-\mathrm{z}$ and $\mathrm{y}-\mathrm{z}$, make a set of three periodic cycles, comprising the PGUs: $\mathrm{x}-\mathrm{y} \equiv \mathrm{U}_{\mathrm{p}}^{\mathrm{o}} ; \mathrm{x}-\mathrm{z} \equiv \mathrm{U}_{\mathrm{p}}^{\prime}$ and $\mathrm{y}-\mathrm{z} \equiv \mathrm{U}_{\mathrm{p}}$; an example is the Pan-STARRS1 Static Sky, New Atlas (2016) which depicts our cosmic space, Obande (2017a).

\section{Symbols}

Figure 6 is an expansion of a segment of Figure 5, observe that:

The figure depicts an envelope of a binary, the twin locates in the adjoining quadrant to form a plane as in Figure 5. Three mutually perpendicular planes intersect at the epicenter to constitute the three PGUs. It explains why 'all particles have charges which are multiples of e-/3', Christianto and Smarandache (2007). Sadly, quantum physics bunches together the three PGUs into a single universe creating untold challenges in an effort to conceptualize nature; for instance, physics is currently at a loss on what to make of indications of observational invisible material world, Clowe et al. (2006), Clarke and Foot (2016), Dokkum et al. (2016).

Key: $\pm \mathrm{X}, \mathrm{A} / \mathrm{B}=$ periodic group, valence or charge; $\mathrm{c}_{\mathrm{o}}=2.997982458 \times 10^{8}$ and $\mathrm{c}^{\mathrm{o}}=3.715352291 \times 10^{-14} \mathrm{rad} \mathrm{s}^{-1}$ transverse vacuum (boson) and matter (fermion) fields respectively - they manifest electrical effects; $\mathrm{v}_{\mathrm{o}}=\pi \mathrm{c}_{\mathrm{o}}=$ $9.418258 \times 10^{8} \mathrm{~m} \mathrm{~s}^{-1}$ and $\mathrm{v}^{0}=\pi \mathrm{c}^{0}=1.167212 \times 10^{-14} \mathrm{~m} \mathrm{~s}^{-1}$, tangential velocities of light in vacuum and in matter respectively - they manifest metric expansion of space and of matter; central open cycle $=$ vacuous core - it manifests atomic nucleus in matter and the black hole in space; the rectangular segment bounded by opposite charges $+\mathrm{xA}$ and $-\mathrm{xB}$ is elaborated in Figure $6 \mathrm{a}$.

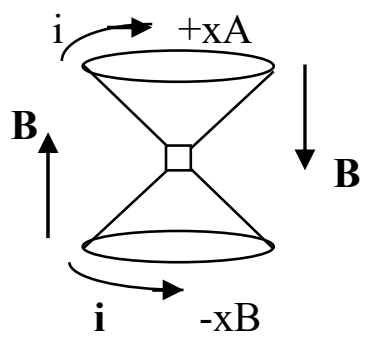

Figure 6a. Sketch of the fundamental periodic quantum envelope 
It comprises an electrical vortical pair inter-twined at the apices; the electro-geometric symmetry (EGM) group or valence, $0, \pm 1 \ldots \pm 4,0$, differs according to arrangement of the pair in a sphere-inscribed cube, Obande $(2017 \mathrm{c})$. We are unable to present the figure here beyond noting that it depicts morphology of the universal energy packet, see for instance, the Warsaw University's 'Hologram of a Single Photon', Wiley Online Library (2016) and notice in Figure $6 a$ the opposing directions of the binding 'magnetic' fields, it is the universal unit mass centripetal acceleration responsible for the (strong) nuclear binding force SNF, $g_{w}=7.943 \times 10^{59} \mathrm{~m} \mathrm{~s}^{-2} \mathrm{~kg}^{-1}, \mathrm{Obande}(2017 \mathrm{~b}$ ).

\section{Disscussion}

\subsection{BH-Motivated Cyclic Spacetime Flux}

On the one hand we have an observational BH process that ionizes neutral matter into charged particles resulting in transformation from visibility to invisibility and on the other we have an unknown invisible process that emits radio waves and neutral atomic and molecular matter from invisible to visible space, Donley et al. (2002), Yale (2006), Tombesi et al. (2015), Leroy et al. (2015), Pan et al. (2015), Das et al. (2015), Dasyra et al. (2015), Whitney (2015), Gallimore et al. (2016), Sanders et al (2016), Oka et al. (2017). Usually, the emission is through galactic nuclei, Richings and Faucher-Giguere (2018), however, off-nucleus evidences exist, Pinto et al. (2016). Were it possible to access invisibility we might find charged particles issuing from visible to invisible space. It suggests that spatial matter emission and 'adsorption' are complementary and opposite sides of a universal cyclic massenergy flux. The reasoning leads to the following key positions regarding the $\mathrm{BH}$ : i) the causal agent that emits matter unto visible space is inverse that which 'meters' ionized particles into invisible space; in other words, conjugate to the observational $\mathrm{BH}$ is an invisible inverse process. With the observational side it ionizes matter into charged particles and channels it into invisible space and on the invisible side it re-composes charged particles into neutral matter and pumps it unto visible space; ii) the BH turns out a multi-faceted device of nature responsible for matter wave frequency modulation, transformation and re-orientation for perpetual circulation of spacetime ST matrices across the four phases of reality to sustain an endless existence; the process is illustrated below in a flowchart.

NASA (2016) groups the BH into two main classes rated here according to electric potential in place of mass: 'low' and 'high' voltage classes, LVC and HVC respectively. Solar mass BH and below classify with the LVC, its process is supposedly much milder than the super-massive BH HVC process; we deduce from Figure 2 and equations (1) and (2) that the LVC handles elements e to $\mathrm{Na}$ while the HVC processes Mg to Am, they both function alike, i.e., matter inter-conversion: visible neutral $\leftrightarrow$ invisible charged particles. Both processes achieve through frequency modulation, transformation and re-orientation, they are complementary and necessarily coincident. We have no further idea of the details beyond literature's observational processes and the present quantitative evidences. However, we think that the observational process can be simulated with a combined frequency modulator/transformer circuitry. Successful simulation of an e-m process that crushes whole solar or galactic system beyond mere dust to charged particles in vacuum space would certainly introduce new dimensions of energy generation and utilization. Notably, the result reveals a curious technology, the BH transforms the atom's visible form into a much higher invisible/dark energy form without changing the chemical character of the element, e.g., in Table 2 iodine: $\mathrm{m}_{\mathrm{p}}^{\mathrm{o}}=0.127 \mathrm{~kg} \mathrm{u}^{-1}, \vartheta_{\mathrm{p}}^{\prime}=2.5 \times 10^{4} \mathrm{~Hz}$ transforms to $\mathrm{m}_{\mathrm{p}}^{\prime}=30.2 \mathrm{~kg} \mathrm{u}^{-1}, \vartheta_{\mathrm{p}}^{\prime}=6.28 \times 10^{7} \mathrm{~Hz}$ but still very much the same element, only invisible in our ref. frame. Furthermore, the process involves considerable energy input yet, it all occurs within an environment of zero Kelvin vacuum field! It suggests an entirely new physics with tremendous potentials. Recall that spontaneous radioactivity begins at At, the element marks NP 9, it is last of nature's periodic cycles $\vartheta_{\mathrm{w}(\mathrm{At})}=7.5 \times 8^{9} \mathrm{~Hz}$; in other words, at the end of its visible cycle the $\mathrm{BH}$, in a yet unknown e-m process that complements radioactivity, returns matter to invisibility for the next round of inter-phase matrix flux cycle. 


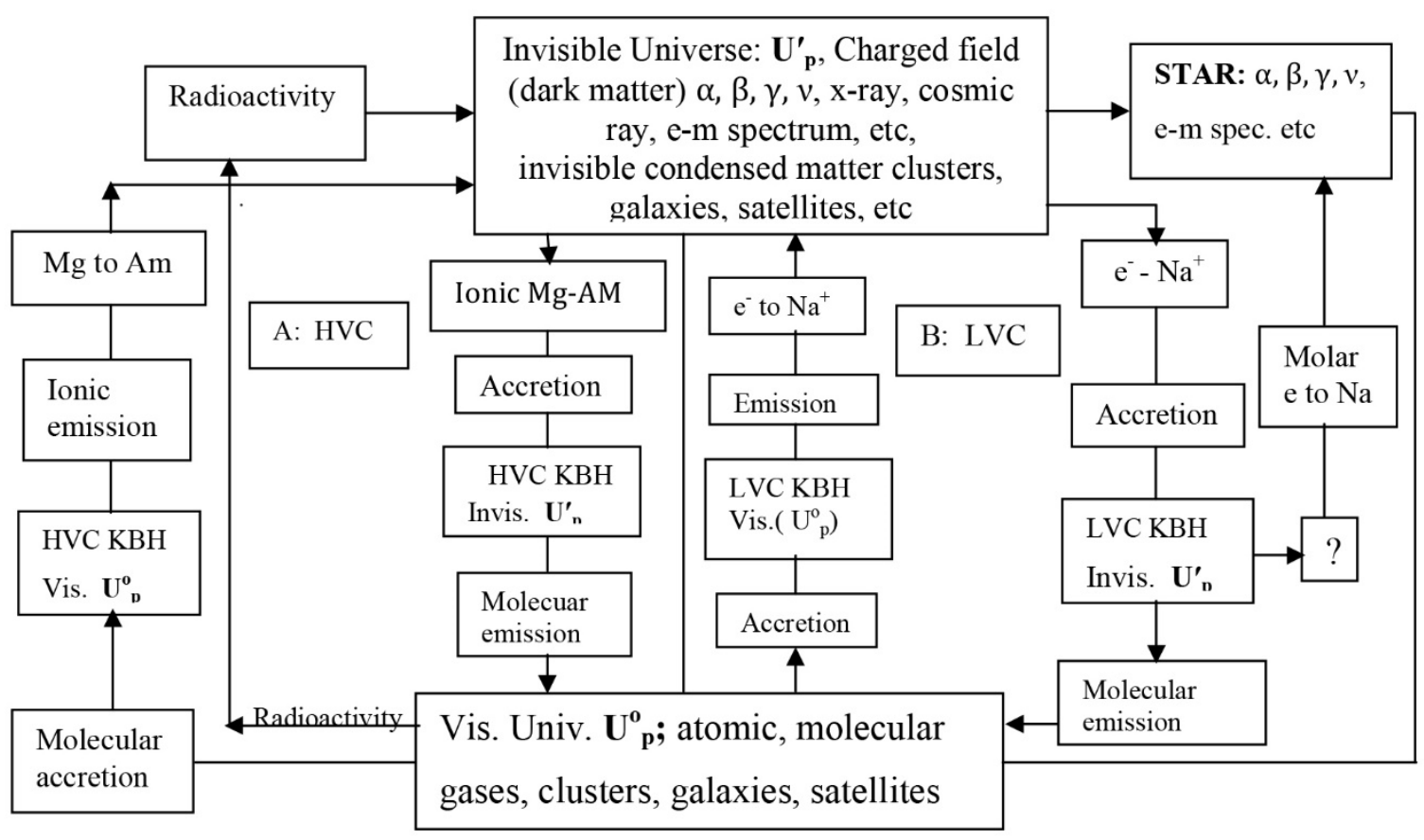

A Flowchart of mass-energy flux exchange across the phases of reality

The position that the BH process is electrodynamics, Miller et al. (2006), Fukumura (2015, 2017), Michili et al. (2018) and not thermodynamics is key to this investigation, it facilitates simulation of the process with a hybrid frequency modulator/transformer model and we hope this very brief account of the process is sufficient to inform the model. The anticipated model is quite simple in principle: input the element's visible frame frequency $\vartheta_{\mathrm{p}}^{\circ}$ and design the circuitry to retrieve its invisible analogue ref. frame's equivalent $\vartheta_{\mathrm{p}}^{\prime}$. Frequency values of elements relevant to the process are presented in Tables 1 and 2; evaluation procedure for $\mathrm{m}_{\mathrm{p}}^{\mathrm{o}}, \mathrm{m}_{\mathrm{p}}^{\prime}, \vartheta_{\mathrm{p}}^{\circ}$ or $\vartheta_{\mathrm{p}}^{\prime}$ with condensed matter's mass-energy relationship $\mathrm{h} 9^{\mathrm{x}} \mathrm{p}=\mathrm{m}_{\mathrm{p}}^{\mathrm{x}} \mathrm{c}^{\mathrm{0} 2}$ where $\mathrm{x}$ refers to the specific PGU has been presented, Obande (2015a).

Table 2. Mass $\mathrm{m} / \mathrm{kg} \mathrm{u}^{-1}$ and frequency $9 / \mathrm{Hz}$ values of some elements in $\mathrm{U}_{\mathrm{p}}^{\mathrm{o}}$ and $\mathrm{U}_{\mathrm{p}}^{\prime}$

\begin{tabular}{lllllll}
\hline Element. & $\mathrm{m}_{\mathrm{p}}^{\mathrm{o}}$ & $\mathrm{m}_{\mathrm{p}}^{\prime}$ & Ratio & $\vartheta_{\mathrm{p}}^{\circ}$ & $\vartheta_{\mathrm{p}}^{\prime}$ & Ratio \\
\hline $\mathrm{E}$ & $9.8 \times 10^{-7}$ & $4.9 \times 10^{-7}$ & 0.5 & 2.034 & 1.017 & 0.5 \\
$\mathrm{C}$ & 0.012 & 0.004 & 0.33 & $2.5 \times 10^{4}$ & $8.35 \times 10^{3}$ & 0.33 \\
$\mathrm{Na}$ & 0.023 & 0.007 & 0.31 & $4.8 \times 10^{4}$ & $1.5 \times 10^{4}$ & 0.31 \\
$\mathrm{Mg}$ & 0.024 & 0.008 & 0.33 & $5.1 \times 10^{4}$ & $1.6 \times 10^{4}$ & 0.31 \\
$\mathrm{I}$ & 0.127 & 30.2 & $1.26 \times 10^{3}$ & $2.5 \times 10^{4}$ & $6.28 \times 10^{7}$ & $2.51 \times 10^{3}$ \\
$\mathrm{Am}$ & 0.234 & $3.1 \times 10^{6}$ & $1.32 \times 10^{7}$ & $5.05 \times 10^{5}$ & $6.55 \times 10^{9}$ & $1.29 \times 10^{4}$ \\
\hline
\end{tabular}

\subsection{Growth}

Periodic (or quantum) mass grows in variable geometric ratio, in terms of $\vartheta$ we have for the chemical element, $\vartheta_{\mathrm{E}}$ $=f \vartheta_{\mathrm{n}}$ where $\vartheta_{\mathrm{n}}=8^{\mathrm{n}}$ for the noble gas, it is the period's fundamental. For the first (inaccessible) noble gas $\vartheta_{1}=8^{1}$ $=8 \mathrm{~Hz}$ (c.f. the octet rule), the value grows exponentially to $\vartheta_{112}=8^{10}=1.073742 \times 10^{9} \mathrm{~Hz}$ for Rn, the last noble gas. The numerical coefficient $f$ increases in arithmetic or geometric progression, the former precedes onset of transition elements (TE), a geometric progression obtains from TE to the end of the periodicity so as to accommodate a doubling of number of elements per period from 8 to 16, see Khazman (2012). Two main observational BH growth processes are recognized: accretion of matter, Volonteri and Rees (2005), O'Leary et al. (2006) Carr et al. (2010), Vesperini et al. (2010), Song et al. (2015), and collision/merger, Zwart et al. (2004), Haehnelt (2005), Cahill (2005), Heckman and Best (2014). Periodicity leaves no room for random accretion and constrains energy packet free roaming; everything, down to the microscopic speck of dust or particulate electron, belongs to a periodic box to which it is gravitationally bound. Similarity with matter suggests bimodal (arithmetic 
and geometric) BH growth processes, it totally rules out random accretion, collision or merger; also, no new spacetime evolution, Avdeenkov and Zloshchastiev (2011), only m-e perpetual re-cycling.

A remarkable similarity between stars and atoms is reported, Oldershaw (2014), it implicates identical mass evolution process across the cosmic mass scale from the atom to the cosmos. Same quantitative expressions should therefore apply to growth rates of atomic and cosmological periodicities. While providing a ready test of the present BH hypothesis, much more importantly, it signals existence of a tremendous potential for observational cosmology with use of the simplest of theoretical analytical tools. Simply, it says that the cosmological parameter $\mathrm{P}_{\text {cosm }}$ should correlate with corresponding atomic parameter $\mathrm{P}_{\text {atom }}$, i.e.,

$$
\mathrm{P}_{\text {cosm }}=\Lambda \mathrm{P}_{\text {atom }}
$$

Thus, $\mathrm{BH}$ and atomic parameters, e.g., $\mathrm{m}_{\mathrm{BH}} / \mathrm{m}_{\mathrm{p}}^{\mathrm{o}}, \mathrm{m}_{\mathrm{BH}} / \vartheta_{\mathrm{p}}^{\circ}, \vartheta_{\mathrm{BH}} / \vartheta^{\circ}$, et cetera, should correlate, where $\mathrm{m}_{\mathrm{p}}^{\mathrm{o}}$ or $\mathrm{m}_{\mathrm{BH}}$; are observational atomic or BH mass; $\vartheta_{\mathrm{p}}^{\circ}$ and $\vartheta_{\mathrm{BH}}$ are the respective frequency values; $\Lambda$ is Oldershaw's parameterspecific scale factor, Oldershaw (1989). Equation (3) should facilitate implementation of a catalogue of maps of complete periodic boxes of the observable universe. A 'complete periodic box' refers to a spatial envelope whose constituent elements duplicate atomic mass evolution of the chemical periodicity from electron to americium, see Obande (2016a). The aligned radio galaxies reported by Taylor and Jagannathan (2015) provide one of the best examples of a complete periodic box; consistent mass of a part, say, bulge or the total galaxy, should correlate with atomic mass of the correspondent chemical element. If verified, the correlation should facilitate symbiotic use of equations (1), (2) and (3) to investigate atomic- and astro-physics.

\subsubsection{Accretion and Size}

Accretion is where theoretical positions meet with severest empirical tests, the subject remains widely open, Miller (2017). Conventional notion holds that the singularity motivates accretion but, classical analysis rules it out. The entire subject of accreting matter and matter that eventually crosses the event horizon into the singularity is pretty fuzzy, so is rationalizing Hawking radiation with the singularity concept, Arp (2005). The classical approach paints a simpler picture.

The classical BH serves solely to inter-convert and re-orient visible and invisible matter in timeless perpetual recycling $\mathrm{U}^{*}{ }_{\mathrm{w}} \leftrightarrow \mathrm{U}^{*}{ }_{\mathrm{p}} \leftrightarrow \mathrm{U}_{\mathrm{p}}^{\mathrm{o}} \leftrightarrow \mathrm{U}_{\mathrm{p}}^{\prime}$. Accretion is not a random event as observation would suggest; a star or some other body does not pass '.. near enough to a massive [or any other type of] black hole to be disrupted', Rees (1988), MacLeod et al. (2013), Piran et al. (2015), Leloudas et al. (2016). Spacetime matrix flux evolves from one periodic group to another just as it does in atomic mass growth. A material of any form, size or ambience, gets accreted when its spatial envelope evolves (progresses) unto group 5 thus, we find evidences for cold and clumpy accretion, Howell (2017), Tremblay et al. (2016), accreting giant stars, MacLeod et al. (2013), decade long accretion or TDE, Lin et al. (2017), changeable accreting materials, La Massa (2016). In principle, an entire galaxy could be the accreting material therefore in time a black hole could grow to the size of an entire galaxy but it would belong to a cluster or universe of immense proportion, a lone $\mathrm{BH}$ would seem an unstable spatial composite much like a pack of chemical radicals.

The subject of BH size is much more involved than observation would suggest and we may not hope to make a reliable inroad until a common formalism informs astrophysics and atomic physics. In principle, there should be a wide spectrum of $\mathrm{BH}$ sizes from those that belong to our visible matrices but exist in hidden (invisible) periodicities, Obande (2016a), to super-giants resulting from mergers; however, conventional Planck size BH, Harada (2006), Carr et al. (2010), is totally ruled out because literature Planck scale neither refers to the atom nor to the 'sub-atomic' particle. A more rigorous analysis will identify the atomic nucleus with the fundamental black hole! See Figure 6a.

\subsection{Alignment}

An observation made with the aid of the India Giant Metrewave Radio Telescope at $612 \mathrm{MHz}$ is reported by Taylor and Jagannathan (2015) as follows: The image contains 65 resolved radio galaxy jets. The spatial distribution reveals a prominent alignment of jet position angles along a "filament" of about $1^{\circ} \ldots$ the probability of chance alignment is less than $0.1 \%$. ... Our results reinforce prior evidence for large-scale spatial alignments of quasar optical polarization position angles. It re-echoes the findings of numerous earlier and more recent reports, Niederste-Ostholt et al. (2010), Crockett (2014). West et al. (2017) propose, as candidate causality, accretion '... along preferred directions that reflect the cosmic web connecting galaxies on large scale [and] ... gravity's relentless tug...', however, Byrd (2017) admits that the effect ' ... remains a mystery.'

An idea of the primitive structure of space is essential for reasonable contemplation of spatial structural alignment. The Big Bang model leaves absolutely no room for a notion of intrinsically structured space and the atomic point- 
mass concept denies the reigning physical paradigm a chance to conjecture nature's primitive form. Our investigation reveals the sphere and the cube as nature's fundamental forms. Depending on what we call 'electrogeometrics 'EGM' dynamics of self-interaction of e-m force fields within the periodic box, Obande (2017c), a wide array of structural morphologies is realizable in matter and in space. A proximate correlation of relative atomic mass with mass number, $\mathrm{m}_{\mathrm{p}}^{\mathrm{x}} / \mathrm{Z}_{\mathrm{n}}$, Khazan (2012), provides an important clue to spatial structural alignment. We showed earlier that a hybrid sphere-inscribed cube is able to account for all matter and spatial morphologies, Obande (2017c), nowhere is this better demonstrated than in spatial structural alignment. Elements of condensed matter periodic cycles are irrevocably diagonally aligned, see: Fig 5, Haehnelt (2005); Figs. 4.71 and 5.1, Nicholas (2012); numerous images of clusters and galaxies, Galaxy (2018), e.g., 'Great Nebula from 1899', NGC4414. The effect is best demonstrated from the object's side view normally associated with 'lenticular galaxy', e.g., NGC 5866, see also NGC 4945; numerous lenticular galaxies oriented in different directions may be seen in the UV Hubble Ultra Deep Field, Teplitz and Rafelski (2014). Elements of the periodic envelope arrange perfectly diagonally and the order is replicated in super-structures that span billions of light years, Crockett (2014). We submit that all galaxies have same intrinsic morphology, the central bulge reinforces the lenticular character of the side view; differences in morphology relate to perspective and age, i.e., the EGM symmetry group.

On the cosmic large-scale, alignment upgrades from linear to honeycomb structures, e.g., distribution of galaxies beyond the Milky Way in 'the panoramic view of the entire infra-red sky', Big Bang (2018). These large-scale structures arrange according to the dictates of what we call EGM symmetry groups SGs, Obande (2017c); the honeycomb structures often favored by nature, Crockett (2014), are SG IV variants, they refer to peak of maturity and progression to NP 5 .

\subsection{Causality of Binaries and Collisions}

Figure 5 is an illustration of the causality of a binary system and eventual collision; it comprises two spatial periodic half-cycles bound together by a common gravitational field generated by the e-m field that defines the two envelopes but mutually repelled by axially aligned e-m polarities. Resultant gravitation and repulsion separates the duo by an equilibrium distance where the opposing forces balance. A member of the duo would appear independent if the couple remained sufficiently distant during the course of progression through nature's periodic groups I to III. However, during this interval, the system goes through noticeable morphological changes characterized by progressive loss of bulk mass common to all material bodies (including humans!), see, e.g., Sciama (1969), it reflects evolution through the four EGMs, Obande (2017c). Separation distance is maintained up to a point at which resultant gravitation overpowers repulsion, the bigger envelope begins to pull the smaller, they may yet retain individual appearances; eventually, however, changes in gross morphology, particularly on the part of the smaller member, become apparent. At group IV the individual envelope has rid itself of significant mass mostly to the gain of the central BH; it may mark onset of neutron star formation and clearer definition of the binary system; angular speed becomes quite significant due primarily to mass loss as the system transits from the margin to the core of group 4, i.e., $+4,0,-4$. At the core there is a sudden total loss of e-m repulsion resulting in gravity-only motivated collision occasioned by total loss of charge, i.e., rotation. The position that collision leads to the next higher BH mass on the logarithmic scale needs be broadened to accommodate the formation of non-rotating neutron star favored by the present hypothesis. Actually, mutual gravitation informs all spatial collisions and a ripe apple's fall is a 'collision' with the Earth as much as the Milky Way might one day collide with Andromeda, so also our cosmic estate with the 'Great Attractor', Stutter (2016); the post collision scenario is still a subject of active investigation. Given the present results showing that the BH is not a hole but an engineered spacetime envelope, the name 'Active Galactic Nucleus' AGN offers a much better description.

It is curious that with $\mathrm{e}=1.0 \mathrm{~Hz}$, the chemical periodicity is comparable to a hierarchical universe of microscopic periodic envelopes (galaxies) that starts with e as unit and Am an ultra-massive galactic composite or cosmos comprising $6.445 \times 10^{9}$ units. Remarkable closeness to the universe's age $\sim 13.7 \times 10^{9}$ years might not be mere coincidence, especially as $2 \mathrm{Am}$ 'galactic' envelopes $=13.89 \times 10^{9}$ units!

\section{Summary and Conclusion}

A classical view of the black hole BH phenomenon is presented. Its positions claim validity with the submission that: reality is field only; the classical and relativistic domains share common quantum characteristics defined by same energy coefficient $\mathrm{h}=6.62607 \times 10^{-34} \mathrm{~J} \mathrm{~s}$ but differ markedly in medium transverse field $\mathrm{c}_{\mathrm{o}}=2.99892458 \mathrm{x}$ $10^{8} \mathrm{rad} \mathrm{s}^{-1}$ and co $=3.71535229 \times 10^{-14} \mathrm{rad} \mathrm{s}^{-1}$ for the boson and the fermion respectively. Common quantization implies common periodicity for vacuum space, the atom, and condensed matter. The approach proceeds to investigate the $\mathrm{BH}$ phenomenon with simple harmonic motion (SHM) formalism and submit as follows: 
Reality is field only and comprises co-existing, mutually interacting 'Absolute' Ref. Frame or Universe, $\mathrm{U}^{*}$, it manifests the cosmic vacuum field; condensed matter Component of the Absolute, $\mathrm{U}^{*}{ }_{\mathrm{p}}$; our Visible Universe $\mathrm{U}_{\mathrm{p}}^{\circ}$; and its Conjugate $\mathrm{U}_{\mathrm{p}}^{\prime}$. The three particulate matter universes manifest observational 'particle-generations'.

As a result of common quantization, classical (Newtonian) physics applies equally to condensed matter and space.

Natural periodicity comprises 9 groups, the 5 th centers all natural quantum (i.e., periodic) envelopes; it identifies with the $\mathrm{BH}$ in space and with the atomic nucleus (i.e., 'gap') in matter.

Far from being a singularity, the BH is an engineered space with multiple functions: on the one hand, it ionizes visible matter to invisible charged particles; on the other, it converts invisible to visible matter and pumps it unto visible space as radiation (notably, radio waves), gaseous, atomic or molecular emission. Hawking's 'apparent horizon' turns out a better description of the BH process than the singularity conjecture.

The visible and invisible BH processes synchronize to ensure sustained infinite m-e cycles - no new creation, only spacetime matrix re-cycling.

The black hole is not a thermodynamics but an electrodynamics process thus, a model comprising a frequency modulator/transformer composite circuitry should provide better description of the process than existing models;

Regardless of size, the $\mathrm{BH}$ is a spacetime matrix flux inflexion zone spanning a significant spatial dimension covering two spatial periods equivalent to conventional periods 2 and 3, i.e., the elements Li to Ar, see Figure 4.

Conjunctive evolution and restriction within the periodic box ensure galaxy-BH mass correlation and question independent $\mathrm{BH}$ growth especially as random inter-box matrix roaming is forbidden.

Theory supports literature's two modes of BH growth - accretion and collision/merger, the former is thought to be intra - while the latter is inter - periodic envelope.

$\mathrm{BH}$ growth is not a random process, matter does not stray near a black hole and gets 'poached'; intra-galactic accretion gives arithmetic (marginal) BH growth, and eventual merger/collision leads to geometric (logarithmic) growth.

Results of an earlier investigation are used to inform that the cube and the sphere are nature's primitive forms, spatial alignment results from diagonal orientation of periodic bodies within cubic spatial envelopes.

All periodic envelopes are binaries, the duo separate by mutual axial projection of like magnetic and electric poles; inter-envelope distance reflects a balance of repulsion and gravitation. Eventual merger results from spontaneous loss of e-m potential upon mutual arrival at group 5 which centers nature's periodicities.

Planck dimensions relatable to reality should read: $\mathrm{M}_{\mathrm{P} \ell}=7.3725 \times 10^{-51} \mathrm{~kg} ; \mathrm{T}_{\mathrm{P} \ell}=1 \mathrm{~s} ; \mathrm{L}_{\mathrm{P} \ell}=\mathrm{c}_{\mathrm{o}} / \mathrm{T}_{\mathrm{P \ell}}=2.99792458 \mathrm{x}$ $10^{8} \mathrm{~m} ; \mathrm{E}_{\mathrm{P} \ell}=6.6261 \times 10^{-34} \mathrm{~J}$ (check: $\mathrm{E}_{\mathrm{P} \ell}=\mathrm{M}_{\mathrm{P} \ell} \times \mathrm{L}_{\mathrm{P} \ell}^{2}$, i.e., $\mathrm{E}=\mathrm{mc}^{2}$ ).

With quantitative evidence that the black hole is not a singularity, the term 'Active Galactic Nucleus' AGN offers much better description of the phenomenon.

In conclusion we notice that observational effects have been interpreted to give the black a crucial role in sustenance of existence. To our best knowledge this is the first attempt to identify the black hole with a role in nature; it offers not only a new idea but, more importantly, a plausible alternative to investigating reality. Admittedly, the approach differs in some respects from established notions but, experience has taught us not to expect radical transformation while insisting on endlessly tailoring belabored approaches. Once again, we conclude with a call, as we often do, to the formidable power of the unimpressive classical mass formula $\mathrm{h} \vartheta=\mathrm{mc}^{2}$ in tackling the challenges of fundamental physics. The possibility to come up with a simpler yet more powerful 'Theory of Everything' is quite remote. The field concept of the BH may be tested as follows: i) accreting and emitting materials should have reversed polarities; ii) galactic mass evolution constituting a complete set in a cluster such as reported for ELAIS N1 by Taylor and Jagannathan should correlate with atomic mass evolution; iii) an appropriately assembled combined modulator/transformer circuitry should provide a superior model of the $\mathrm{BH}$ process than thermodynamics.

\section{References}

Arp, H. (2005). Observational Cosmology: From High Redshirt Galaxies to the Blue Pacific. Prog. Phys., 3, 3.

Avdeenkov, A. V., \& Zloshchastiev, K. G. (2011). Quantum Bose liquid with nonlinearity: Self-sustainability and emergence of spatial extent. Retrieved from https://arxiv.org/pdf/1108.0847..pdf

Big Bang. (2018). In Wikipedia, the free encyclopedia. Retrieved from https://en.wikipedia.org/wiki/BigBang

Born, M. (1935). The Mysterious Number. Retrieved from http://www-old.ias.ac.in/j_archive/proca/2/top.html 
Byrd, D. (2017). Galaxies have aligned for 10 billion years. Retrieved from https://earthsky.com/space/galaxieshave-aligned-for-10-billlion-years

Cahill, R. T. (2005). Black Holes in Elliptical and Spiral Galaxies and in Globular Clusters. Prog. Phys., 3, 51.

Carr, B. J., Kohri, K., Sendouda, Y., \& Yokoyama, J. I. (2010). New cosmological constraints on primordial black holes. Physical Review D, 81(10), 104019.

Christianto, V., \& Smarandache, F. (2007). Thirty Unsolved Problems in the Physics of Elementary Particles. Prog. Phys., 4, 112

Clarke, J. D., \& Foot, R. (2016). Plasma dark matter direct detection. Journal of Cosmology and Astroparticle Physics, 2016(01), 029.

Clowe, D., Bradac, M., \& Gonzallez, A. H. (2006). A Direct Evidence of the Existence of Dark Matter. Retrieved from https://arxiv.org/abs/astro-ph/0608407

Conselice, C. J., Wilkinson, A., Duncan, K., \& Mortlock, A. (2016). The evolution of galaxy number density at $\mathrm{z}<8$ and its implications. The Astrophysical Journal, 830(2), 83.

Consiglio, J. (2015). How and Why the Universe is Natural. Appl. Phys. Res., 7(3), 69. https://dx.doi.org/10.5539/ apr.v7n3p69

Consiglio, J. (2016). On Quantization and Resonance Paths. Prog. Phys., 12(3), 259.

Crockett, C. (2014). Galaxies may be aligned across 1 billion light-years. Science News, 186(13), 6-6.

Das, M., Saito, T., Iono, D., Honey, M., \& Ramya, S. (2015). Detection of molecular gas in void galaxies: implications for star formation in isolated environments. The Astrophysical Journal, 815(1), 40. https://doi.org./10.1088/0004-673X/815/1/40

Dasyra, K. M., Bostrom, A. C., Combes, F., \& Vlahakis, N. (2015). A radio jet drives a molecular and atomic gas outflow in multiple regions within one square kiloparsec of the nucleus of the nearby galaxy IC5063. The Astrophysical Journal, 815(1), 34. https://doi.org/10.1088/0004-637X/815/1/34

Donley, J. L., Brandt, W. N., Eracleous, M., \& Boller, T. (2002). Large-amplitude X-ray outbursts from galactic nuclei: a systematic survey using ROSAT archival data. The Astronomical Journal, 124(3), 1308.

Fukumura, K., Kazanas, D., Shrader, C., Behar, E., Tombesi, F., \& Contopoulos, I. (2017). Magnetic origin of black hole winds across the mass scale. Nature Astronomy, 1(4), 0062.

Fukumura, K., Tombesi, F., Kazanas, D., Shrader, C., Behar, E., \& Contopoulos, I. (2015). Magnetically driven accretion disk winds and ultra-fast outflows in PG 1211+ 143. The Astrophysical Journal, 805(1), 17. https://10.1088/0004-637X/805/1/17

Galaxy. (2018). In Wikipedia, the free encyclopedia. Retrieved from https://en.wikipedia.org/wiki/Galaxy

Gallimore, J. F., Elizur, M., \& Maialino, R. (2016). Bipolar Molecular Emission from an AGN Torus. Retrieved from https://arxiv.org/pdf/16o8.02210v1.pdf

Haehnelt, M. G. (2005). The connection between the formation of galaxies and that of their central supermassive black holes. Philosophical Transactions of the Royal Society of London A: Mathematical, Physical and Engineering Sciences, 363(1828), 705-713.

Harada, T. (2006). Is there a black hole minimum mass?. Physical Review D, 74(8), 084004.

Hawking, S. W. (2014). Information preservation and weather forecasting for black holes. arXiv preprint arXiv:1401.5761.

Heckman, T. M., \& Best, P. N. (2014). The coevolution of galaxies and supermassive black holes: insights from surveys of the contemporary universe. Annual Review of Astronomy and Astrophysics, 52, 589-660. https://doi.org/10.1146/annurefastro-081913-05722

Hobson, A. (2013). There are no particles, there are only fields. American Journal of Physics, 81(3), 211-223.

Howell, E. (2017). Winds from monster black holes can rapidly change their temperature. Retrieved from https://www.foxnews.com/sscience/2017/03/03/wnds-from-monster-black-holes-can-rapidly-change-their temp.html

Khazman, A. (2012). The Upper Limit of the Periodic Table of Elements Points Out to the "Long" Version of the Table, Instead of the "Short". Prog. Phys., 1, 45. 
La Massa, S. (2016). A black hole changes its feeding habits. Nature, 540, 48. https://doi.10.1038/nature 20480

Leloudas, G., Fraser, M., Stone, N. C., van Velzen, S., Jonker, P. G., Arcavi, I., ... \& Miller-Jones, J. C. A. (2017). The superluminous transient ASASSN-15lh as a tidal disruption event from a Kerr black hole. Nature Astronomy, 1(1), 0002. https://doi.org/10.1038/s41550-016-0002

Leroy, A. K., Walter, F., Martini, P., Roussel, H., Sandstrom, K., Ott, J., ... \& Dessauges-Zavadsky, M. (2015). The Multi-phase Cold Fountain in M82 Revealed by a Wide, Sensitive Map of the Molecular Interstellar Medium. The Astrophysical Journal, 814(2), 83. https://doi.org/10.1088/0004-637X/814/2/83

Lin, D., Guillochon, J., Komossa, S., Ramirez-Ruiz, E., Irwin, J. A., Maksym, W. P., ... \& Zauderer, B. A. (2017). A likely decade-long sustained tidal disruption event. Nature Astronomy, 1(3), 0033.

Macken, J. (2011). The Universe is only Spacetime. Retrieved from http://onlyspacetime.com

MacLeod, M., Ramirez-Ruiz, E., Grady, S., \& Guillochon, J. (2013). Spoon-Feeding Giant Stars to Supermassive Black Holes: Episodic Mass Transfer from Evolving Stars and their Contribution to the Quiescent Activity of Galactic Nuclei. The Astrophysical Journal, 777(2), 133.

McConnell, N. J. (2012). Black Hole Masses in Nearby Brightest Cluster Galaxies (Doctoral dissertation, UC Berkeley).

Michilli, D., Seymour, A., Hessels, J. W. T., Spitler, L. G., Gajjar, V., Archibald, A. M., ... \& Heald, G. H. (2018). An extreme magneto-ionic environment associated with the fast radio burst source FRB 121102. Nature, 553(7687), 182. https://doi.org/10.1038/nature25149

Miller, J. (2017). Accretion physics: It's not U, it's B. Nature Astronomy, 1(4), 0070. https://doi.org/10.1038/ s41550-017-0070

Miller, J. M., Raymond, J., Fabian, A., Steeghs, D., Homan, J., Reynolds, C., ... \& Wijnands, R. (2006). The magnetic nature of disk accretion onto black holes. Nature, 441(7096), 953.

NASA. (2016). Black Holes. Retrieved from https://science.nasa.gov/astrophysics/focus/black-holes-babies-andgiants

New Atlas. (2016). Static Universe. Retrieved from https://newatlas.com/pan-starrs-largest-digital-survey-visibleuniverse

Niederste-Ostholt, M., Strauss, M. A., Dong, F., Koester, B. P., \& McKay, T. A. (2010). Alignment of brightest cluster galaxies with their host clusters. Monthly Notices of the Royal Astronomical Society, 405(3), 20232036. https://doi.org/10.1111/j.1365-2966.2010.16597.x

O’leary, R. M., Rasio, F. A., Fregeau, J. M., Ivanova, N., \& O’Shaughnessy, R. (2006). Binary mergers and growth of black holes in dense star clusters. The Astrophysical Journal, 637(2), 937.

Obande, O. P. (2013). Notes on Russellian Cosmogony-Part 1: Absolute Atomic Mass. The Int. J. Engnr. \& Sci, 2(4), 68-77.

Obande, O. P. (2015a). Notes on Russellian cosmogony. II. A procedure for theoretical calculation of relative atomic mass and internal energy. Phys. Essays, 28(1), 78. https://doi..org/10.4006/0836-1398-28.1.78

Obande, O. P. (2015b). Classical mechanics analysis of the atomic wave and particulate forms. Int. J. Eng. Sci., $4(6), 1$.

Obande, O. P. (2016a). Atomic Mass: Origin, Units and Constants. Appl. Phys. Res., 8(1), 92. https://doi.org/10.5539/apr.v8n1p92

Obande, O. P. (2016b). On the Photon's Identity: Implications for Relativity and Cosmology. Appl. Phys. Res., $8(5), 1$ https://doi.org/10.5539/apr.v8n5p1

Obande, O. P. (2017a). The Pan-STARRS1 Static Sky: A Classical Physics Goldmine for Cosmology, Astrophysics and Atomic Physics. Appl. Phys. Res., 9(5), 75. https://doi.org/105539/apr.v9n6p75

Obande, O. P. (2017b). On the Fundamental Physical Constants: I. Phenomenology. Appl. Phys. Res., 9(5), 42. https://doi.org/10.5539/apr.v9n5p42

Obande, O. P. (2017c). On the Fundamental Physical Constants: II. Field Coupling geometry. Appl. Phys. Res., 9(5), 62. https://doi.org/10.5539/apr.v9n5p62 
Oka, T., Tsujimoto, S., Iwata, Y., Nomura, M., \& Takekawa, S. (2017). Millimetre-wave emission from an intermediate-mass black hole candidate in the Milky Way. Nature Astronomy, l(10), 709. https://doi.org/10. 1038/s41550-017-02442-2

Oldershaw, R. L. (1989). Self-Similar Cosmological Model: Introduction and Empirical Tests. Retrieved from https://www3.amherst.edu/ rloldershaw/OBS.HTML

Oldershaw, R. L. (2003). An Apparent Gap In Stellar Mass Distributions At 0.7M@ And A Possible Explanation. Retrieved from https://www3.amherst.edu/ rlodershaw/smf.html

Oldershaw, R. L. (2014). A Surprising Similarity Between Stars and Atoms. Retrieved from https://www3. amherst.edu/ rloldershaw/stars2/isomorphism.html

Pan, H. A., Kuno, N., Koda, J., Hirota, A., Sorai, K., \& Kaneko, H. (2015). Molecular Gas and Star-formation Properties in the Central and Bar Regions of NGC 6946. The Astrophysical Journal, 815(1), 59. https://doi.org/10.1088/0004-673X/1/59

Pinto, C., Middleton, M. J., \& Fabian, A. C. (2016). Resolved atomic lines reveal outflows in two ultraluminous X-ray sources. Nature, 533(7601), 64.

Piran, T., Svirski, G., \& Krelik, J. (2015). “Circulation” vs. Accretion - What Powers Tidal Disruption Events?. Retrieved from https://arxiv.org/pdf/1502.05792.pdf

Rafelski, M. (2018, January). The Ultraviolet Hubble Ultra Deep Field. In American Astronomical Society Meeting Abstracts (Vol. 231).

Rees, M. J. (1988). Tidal disruption of stars of 106 - 108 solar masses in nearby galaxies. Nature, 333, 523.

Reines, A. E., \& Volonteri, M. (2015). Relations between central black hole mass and total galaxy stellar mass in the local universe. The Astrophysical Journal, 813(2), 82.

Richings, A. J., \& Faucher-Giguere, C. A. (2017). The origin of fast molecular outflows in quasars: molecule formation in AGN-driven galactic winds. Monthly Notices of the Royal Astronomical Society, 474(3), 36733699. https://doi.org/10.1093mnras/stx3014

Sanders, J. S., Fabian, A. C., Taylor, G. B., Russell, H. R., Blundell, K. M., Canning, R. E. A., ... \& Grimes, C. K. (2016). A very deep Chandra view of metals, sloshing and feedback in the Centaurus cluster of galaxies. Monthly Notices of the Royal Astronomical Society, 457(1), 82-109.

Sciama, D. W. (1969). Is the Galaxy losing Mass on a Time Scale of a Billion Years?. Nature, 224(5226), 1263. https://doi.org/10:1038/2241263a0

Song, C. Y., Liu, T., Gu, W. M., Hou, S. J., Tian, J. X., \& Lu, J. F. (2015). Evolutions of stellar-mass black hole hyperaccretion systems in the center of gamma-ray bursts. The Astrophysical Journal, 815(1), 54. https://doi.org/10.1088/0004-637X/815/1/5

Stutter, P. (2016). Will the Great Attractor Destroy Us?. Retrieved from https://www.space.com/33579-will-thegreat-attractor-destroy-us.html

Taylor, A. R., \& Jagannathan, P. (2016). Alignments of radio galaxies in deep radio imaging of ELAIS N1. Monthly Notices of the Royal Astronomical Society: Letters, 459(1), L36-L40.

Tombesi, F., Meléndez, M., Veilleux, S., Reeves, J. N., González-Alfonso, E., \& Reynolds, C. S. (2015). Wind from the black-hole accretion disk driving a molecular outflow in an active galaxy. Nature, 519(7544), 436. https://doi.org/10.1038/nature14261

Tremblay, G. R., Oonk, J. R., Combes, F., Salomé, P., O’Dea, C. P., Baum, S. A., ... \& McDonald, M. A. (2016). Cold, clumpy accretion onto an active supermassive black hole. Nature, 534(7606), 218.

Van Dokkum, P., Abraham, R., Brodie, J., Conroy, C., Danieli, S., Merritt, A., ... \& Zhang, J. (2016). A high stellar velocity dispersion and $\sim 100$ globular clusters for the ultra-diffuse Galaxy dragonfly 44 . The Astrophysical Journal Letters, 828(1), L6.

Vesperini, E., McMillan, S. L., D'Ercole, A., \& D'Antona, F. (2010). Intermediate-Mass Black Holes in Early Globular Clusters. The Astrophysical Journal Letters, 713(1), L41.

Volonteri, M., \& Rees, M. J. (2005). Rapid growth of high-redshift black holes. The Astrophysical Journal, 633(2), 624. 
Warsaw University. (2016). Hologram of a Single Photon. Retrieved from https://wileyonlinelibrary.wiley.com/ doi/pdf/10.1002/opph.201690044

West, M. J., De Propris, R., Bremer, M. N., \& Phillipps, S. (2017). Ten billion years of brightest cluster galaxy alignments. Nature Astronomy, 1(7), 0157.

Whitney, C. (2015). Black Hole Has a Major Flare. Retrieved from https://www.jpl.nasa.gov/news/ news.php? feature $=4753$

Yale University. (2006). Evidence for Ultra-Energetic Particles in Jet from Black Hole. Retrieved from https://www.yale.edu/opa/news/06-06-20-01.all.html

Zwart, S. F. P., Baumgardt, H., Hut, P., Makino, J., \& McMillan, S. L. (2004). Formation of massive black holes through runaway collisions in dense young star clusters. Nature, 428(6984), 724.

\section{Copyrights}

Copyright for this article is retained by the author(s), with first publication rights granted to the journal.

This is an open-access article distributed under the terms and conditions of the Creative Commons Attribution license (http://creativecommons.org/licenses/by/4.0/). 